\title{
TIPS AND TRICKS FOR CHARACTERIZING SHAPE MEMORY ALLOY WIRE: PART 2-FUNDAMENTAL ISOTHERMAL RESPONSES
}

\author{
by C.B. Churchill, J.A. Shaw, and M.A. Iadicola
}

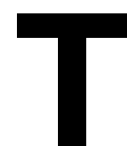

his is the second in a series of articles to introduce phenomena and experimental subtleties that can lead to difficulties in testing shape memory alloy (SMA) wire. Our aims were to acquaint uninitiated engineers with SMA testing and to highlight pitfalls in technique and interpreting test results. In the previous article (Part 1), ${ }^{1}$ thermomechanical experiments were presented on two commercial Nitinol alloys: a shape memory (SM) wire, having an austenite finish temperature, $A_{\mathrm{f}}$, above room temperature, and a superelastic (SE) wire, having an $A_{\mathrm{f}}$ below room temperature. Both SM and SE behavior were demonstrated for each in different temperature regimes along with a description of the underlying microstructural mechanisms responsible for these unusual behaviors. Differential scanning calorimetry (DSC) thermograms were provided for each alloy as a first step to quantify their transformation temperatures, specific heats, and latent heats of transformation.

In this article (Part 2), we discuss techniques and good practice for temperature control and strain measurement. The characterization of the same two alloys is continued through a series of isothermal experiments to show the dramatic range of tensile responses in a temperature window spanning the respective stress-free transformation temperatures. Characteristic transformation stresses and strains are mapped to quantify the thermomechanical sensitivities in the material. Last, material instabilities, strain localization, and propagating transformation fronts are discussed along with their implications on measured responses.

\section{UNIAXIAL EXPERIMENTAL METHODS}

Before presenting experimental data, this section provides an overview of different methods for thermomechanical testing of SMA wire. The section discusses the trade-offs associated with the choice of loading method, strain control/measurement, and temperature control/measurement.

\section{Loading Method}

Once DSC scans have been performed to establish the relevant transformation temperatures (as discussed previously

Editor's Note: This ET feature series is intended as an introduction to this exciting area of experimental mechanics. It aims to increase awareness of active materials and to promote their consistent characterization by disseminating best practices from leading researchers in the field. Each article in the series will address the characterization of one commercially significant active material. Series editors: Nilesh D. Mankame and Paul W. Alexander.

C.B. Churchill (churchc@umich.edu) is a graduate student and J.A. Shaw is an associate professor with the Department of Aerospace Engineering, University of Michigan, Ann Arbor, MI. M.A. Iadicola is a staff scientist in the Metallurgy Division of the National Institute of Standards and Technology, Gaithersburg, MD. in Part $1^{1}$ ), it is necessary to obtain an overview of the material behavior with respect to axial load-elongation-temperature space. A clear picture of the thermomechanical behavior of an SMA requires multiple experiments over a wide range of temperatures. Mechanical data at one or even two temperatures are not generally sufficient due to the material's extreme temperature sensitivity, as will be demonstrated in the "Isothermal Mechanical Responses" section. Thermomechanical data are customarily obtained in one of two methods: either (1) holding constant axial load and sweeping temperature up and down while measuring the strain response, or (2) holding constant temperature and sweeping the elongation up and down while measuring the axial load response.

The first method can be executed by hanging a weight on the wire or using a conventional testing machine in load control, a so-called soft loading device. A servo-hydraulic testing machine most easily accomplishes the task, although an electromechanical testing machine may be used if a suitable feedback control is available to simulate load control. Several temperature scans at different load levels are required to obtain a view of the phase transformation "surfaces" in force-elongation-temperature space. Depending on the setup, it may be difficult to precisely control the temperature rate, and thermal inertia of the setup may limit it to inconveniently slow rates.

The second method is most easily executed with a screwtype electromechanical testing machine in displacement control, a so-called stiff loading device, although it can also be accomplished using elongation-control feedback. The control loop in the machine must be carefully tuned to the stiffness of the test specimen being used. We generally prefer the stiff loading device (isothermal mode) for SMA constitutive experiments since it tends to be more well behaved and reveals more detailed behavior if instabilities occur in the material, as discussed further in the "Material Instability and Transformation Fronts" section. The material behavior is history dependent (hysteretic), and for now, we focus on the monotonic load-unload behavior across the strain range where obvious phase transformation occurs, that is, we are interested in the so-called "outer loops" of the behavior.

\section{Strain Measurement and Control}

The next issue the experimentalist faces is the choice of strain measurement and control, a choice made especially important by peculiarities in SMA behavior. Here, we outline some common choices progressing from simple to complex. 


\section{Global Strain Measurement}

The simplest strain measurement can be derived from the displacement of the testing machine's crosshead $(\delta)$, thereby giving a "global," or average, strain measure $\delta / L$, where $L$ is the free length of the wire specimen between the grips. This works well, provided the stackup of fixtures between the base and the crosshead is much stiffer than the test specimen. While grip displacement provides the preferred control method (as discussed later), it may not give the best strain measurement due to stress/strain concentrations induced at the grips and possible grip slippage.

\section{Local Strain Measurement}

It is of course good practice to measure the "local strain" in the gage section of a test specimen for accurate strain measurement, and SMA testing is no exception. The need is obvious for standard "dog-bone" specimens, which are thicker at the ends and taper to a uniform middle gage section, since strain gradients across the tapered region and strains measured from grip displacement will not match the average strain in the gage section. The need may be less obvious for straight wire specimens, but it is still necessary to avoid grip artifacts in the strain measurement and to capture possible strain localization, as explained in "Material Instability and Transformation Fronts" section.

- Strain gages: While strain gaging is commonly used to accurately measure local strains in the gage section of conventional specimens, it is usually not a practical option for SMA wire. Attaching strain gages to very thin wire specimens is difficult, and most strain gages are incapable of withstanding strains to $\approx 8 \%$ and back without suffering plastic deformation.

- Mechanical extensometer: Alternatively, a clip-on extensometer can be easily installed and can follow axial strains of up to $20 \%$ or larger, depending on the model. They provide accuracy near to that of strain gages. One must ensure, however, that the weight of the extensometer, which is attached at two knife-edges and cantilevered from the specimen, does not induce any significant bending of the wire specimen. For very thin wire specimens, it may be necessary to counterbalance the extensometer so that it "floats" aside the specimen.

- Video extensometer: A noncontacting extensometer is another instrument that can be used to virtually eliminate any loading artifacts that might arise from mechanical extensometry or strain gaging, which is especially useful for testing very compliant specimens. An optical video extensometer is a noncontacting measurement system that uses targets illuminated by visible light. A highresolution digital camera takes images of the specimen, while a computer processes the images in real time, noting the distance between two or more visible markers. These may be tags affixed to the specimen or painted markers. Fairly inexpensive systems (about $\$ 10,000$ ) can be used when the strains are quite large, such as when testing elastomeric specimens. These have not been typically used for stiff metallic specimens where the strains are relatively small, but more expensive systems $(\$ 25,000$ and more) are now available that provide sufficient accuracy at small strains (resolutions down to $0.50 \mu \mathrm{m})$ and high frame rates (above $200 \mathrm{kHz}$ ).
- Laser extensometer: Another noncontacting version of the above concept is a laser extensometer system (we use model EIC-05 from Electronics Research Corp., Irwin, PA), where thin retroreflective tags are affixed to the specimen and a laser sheet is shined across the tags. (Certain commercial equipment, instruments, or materials are identified in this article to foster understanding. Such identification does not imply recommendation or endorsement by the authors or their employers, nor does it imply that the materials or equipment identified are necessarily the best available for the purpose.) The system in turn detects the reflected laser signal from the tags and gives an accurate measurement of the distance between the edges of the tags $\left(L_{\mathrm{e}}\right)$. The resolution of our system is about 2 $\mu \mathrm{m}$, so the accuracy of the length measurement is mostly dependent on the quality of the tag attachment and alignment of the laser sheet with the specimen axis. Overall, the strain accuracy is nearly as good as a mechanical extensometer, and it can be used to very large strains (we even use it for elastomeric testing to several $100 \%$ strain). It also circumvents the need to attach any bulky instrumentation to the specimen, which might prevent other types of imaging, and adds essentially no additional loads or moments, which are especially useful for testing very thin wire. This requires a line of sight between the laser system and the specimen, and if the laser sheet is planar (as ours is), it can be used through a window of an air chamber without any parallax concerns. Our system can be used at rates up to $100 \mathrm{~Hz}$, but we find the signal to be much less noisy by oversampling in time, effectively limiting the measurement rate to about $20 \mathrm{~Hz}$. This is sufficient for slow to moderate strain rates of typical wire SMA specimens.

\section{Full-Field Strain Measurement}

The above methods provide strain measurements of specific regions of interest in a specimen defined by the locations and spacing of tags/markers/knife-edges. The methods are discrete in nature, giving strain measurements necessarily averaged across a region of interest. Here, we mention two full-field strain measurement techniques that give a broader view of the strain field in the specimen.

- Brittle coatings: A classical technique for full-field strain measurement is to coat the specimen with a brittle coating that changes its reflectivity as it is strained and to then take photographs of the specimen during testing. The optical characteristics of the coating can be calibrated to known strains, but the accuracy is usually limited. The information achieved is full field in nature but is fairly qualitative and can only be used for one loading cycle without reapplication since the brittle coating is usually permanently deformed at typical SMA strains. In fact, certain Nitinol alloys have a naturally occurring dark oxide layer that can sometimes be used in this way to track nonuniform strain fields. ${ }^{2}$

- Digital image correlation: A more quantitative technique involves digital image correlation, where digital images are taken during testing and then postprocessed to give the displacement and strain fields along the specimen. ${ }^{3}$ The resolution and accuracy of the measurement are dependent on the details of the specimen surface specularity, 
which may require the specimen surface to be treated/painted to give good results (requiring some art). This is the most elaborate technique mentioned here, and we include it only for completeness, yet if well calibrated, it can provide a wealth of deformation information, especially when strain fields are nonuniform and deformations are multiaxial. ${ }^{4}$

\section{Gripping Issues}

A few words should be said about gripping wire specimens. In the setups to be presented here, we used flat, hardened steel plates, each having a shallow v-groove to aid in specimen installation and alignment. In setups where the specimen was immersed in a fluid bath, the plates were simply clamped together by cap screws. This was adequate in most cases, but high torques were required to prevent excessive slippage at the highest loads. In setups in an air chamber, pneumatic grips were used to maintain constant pressure between the grip plates. This worked better since the clamping force was held constant even as the specimen strained and thinned across the diameter. Nevertheless, some amount of grip slippage is nearly unavoidable when clamping wire in this manner for additional reasons (which will be discussed in the "Material Instability and Transformation Fronts" section). Spool grips are also available from various testing machine manufacturers, which nearly eliminate any stress concentrations at the ends. This is a useful technique if one simply wants to find the breaking strength of the wire, where stress concentration could otherwise cause premature failure. We do not, however, recommend them for careful experiments for constitutive characterization since the effective free length of the specimen remains uncertain due to friction effects on the spool.

\section{Strain Control}

In the experiments to be presented, the specimen elongation, $\delta$, was controlled at a prescribed rate $(\dot{\delta})$ during loading $(\dot{\delta}>0)$ and unloading $(\dot{\delta}<0)$, chosen in the range of global strain rates $\dot{\delta} / L= \pm 5 \times 10^{-5} \mathrm{~s}^{-1}$ to $\dot{\delta} / L= \pm 4 \times 10^{-4} s^{-1}$. These strain rates might seem excessively slow to those familiar with mechanical testing of conventional metal (about $\dot{\delta} / L= \pm 1 \times 10^{-3} s^{-1}$ ) but are in fact necessary to achieve near-isothermal conditions for SMA wire (as will be explained in the next article in this series).

As a final caution, one must resist the temptation to use a "strain control" mode via a local strain measurement (such as extensometer) feedback due to possible strain localization in SMAs as discussed further in the "Material Instability and Transformation Fronts" section. When using a stiff loading device, like a screw-type electromechanical testing machine, the imposed end displacement could wander while straining occurs outside the extensometer region. More dangerously still, when using a soft loading device, like a servo-hydraulic testing machine, this control mode could cause the machine to jump suddenly if no straining is detected within the extensometer region. Instead, grip displacement (global strain control) should be used as the control mode.

\section{Temperature Control and Measurement}

The next consideration is the choice of method for temperature control and measurement of the specimen, and several are detailed below. Unlike conventional metals, where moderate changes in temperature can be tolerated without affecting the quality of the results, a few degrees change in specimen temperature can significantly influence the results of an SMA experiment, so more care is typically needed.

- Joule heating: Electrical ohmic (Joule) heating is a common method for changing the temperature of an SMA wire during testing, and it is often used for actuation in devices. The method is simple and convenient but has several limitations. It is typically only used for SM wire (i.e., those with sufficiently high transformation temperatures) for testing in room temperature air since the ambient temperature must be sufficiently low compared to the material's transformation temperatures. The electrical power and convective environment must be carefully controlled since the material's electrical resistivity is somewhat phase dependent ${ }^{5}$ and stray air currents can cause undesirable fluctuations in the test specimen's temperature. Even seasonal changes and heating/air-conditioning systems in the laboratory can affect these results, so a suitable enclosure is often desirable. The electrical attachment method can also be problematic since any contact resistance may be of the same order as the specimen resistance. Furthermore, temperature measurement is difficult since thermocouples attached to the SMA wire specimen give unreliable measurements when large electrical current is flowing through the specimen. A noncontacting infrared temperature probe can potentially be used, but this takes some care to get accurate temperature measurements from thin wire specimens.

- Environmental chamber: Another common method is to use a temperature-controlled air chamber fitted within a mechanical testing frame. If it is available with a cooling system, such as liquid $\mathrm{CO}_{2}$ or liquid $\mathrm{N}_{2}$, the temperature range for testing is considerably broadened into subambient temperatures, accommodating testing of SE SMA wire across its range of transformation temperatures. Thermocouples can be attached directly to the test specimen to monitor the local temperature in the gage section, but the thermocouple wire should be relatively fine compared to the SMA wire diameter to achieve accurate temperature measurement. We commonly use $0.076-\mathrm{mm}$ (0.003 inch)-diameter exposed junction K-type thermocouples attached to the SMA specimen by small clips. A daub of thermally conductive paste (such as Omegatherm 201) ensures good thermal contact between the thermocouple junction and the specimen surface. We recommend additional thermocouples be used to monitor the air temperature in proximity to the specimen, as well as the temperature of grips that are typically massive compared to the test specimen and have significant thermal lag. Infrared temperature measurement is usually not practical because typical window glass is opaque to infrared radiation. Overall, the advantage of the air chamber method is the flexibility in ambient temperature for testing, but stray air currents within typical commercial chambers can still cause temperature fluctuations, especially at subambient temperatures where forcedair cooling is used (typically $\pm 5^{\circ} \mathrm{C}$ ). We found that attaching a simple flow diffuser to the back of our 
chamber at the forced-air exit significantly improved the temperature spatial and temporal uniformity to about $\pm 1^{\circ} \mathrm{C}$.

- Liquid bath: One of the most precise temperature control methods involves immersing the test specimen in a liquid bath, such as water, that is temperature controlled by an external fluid circulator. This advantage comes with some additional complexity and typically requires a custom-made experimental setup. The temperature range for testing is limited by the freezing/boiling points of the working fluid, but many suitable fluids can be selected based on the known transformation temperatures of the SMA specimen to be tested. Thermocouples can still be used for measurement of fluid temperature and specimen temperature, provided they have been waterproofed (or are compatible with the chosen working fluid). Mechanical extensometers for strain measurement are typically not waterproof, so some suitable modification or coating addition is required to electrically insulate them. Infrared temperature measurement is not possible since most liquids are opaque to infrared radiation.

- Conduction contact: The last temperature control method to mention is to use a temperature-controlled heat sink in thermal contact with the back side of the SMA wire specimen. ${ }^{6}$ Potentially, this can result in an even more precise control of the SMA specimen temperature, provided the specimen diameter is relatively small so that temperature gradients across the diameter can be reasonably neglected (which is often the case). In this scheme, a thermal grease can be used to ensure good thermal contact while allowing the specimen to slide freely in its axial direction. Of course, careful alignment of the experimental stackup is required to make this work well. We also found that inserting thermocouple wafers between the heat sink and the specimen allows fine control of the temperature, and using multiple wafers along the specimen length can even allow control of nonuniform temperature distributions (that are useful in some cases, as discussed in a later article of this series). Another important advantage of this scheme is that it leaves the entire front surface of the specimen free for full-field imaging, both optical (strain) and infrared (temperature) measurements.

- Infrared thermography: If available, a digital infrared radiometer (thermal imaging system) can give full-field surface temperature information that is especially useful in interpreting experimental results. Typical research radiometers detect temperature changes on the order of $0.1^{\circ} \mathrm{C}$ and have array sizes between $256 \times 256$ and $1024 \times 1024$. If the wire has a sufficiently small diameter, the measured surface temperature is nearly the same as the temperature at the core of the wire (which can be verified by calculating the Biot number; see Iadicola and Shaw $\left.{ }^{7}\right)$. The most accurate temperature results with infrared thermography are obtained when the specimen surface has an emissivity near unity (ideal blackbody) and the surface is flat. Nitinol, however, can be obtained in a variety of surface conditions from heavily oxidized (black) to moderately oxidized (coppery) to highly polished (shiny), that is, with a large range of emissivities. Since accurate temperature measurement requires a sufficiently high emissivity (low reflectivity) of the wire surface, it may be necessary to paint the wire to raise its emissivity above 0.8 , generally a good goal. The curved surface of the wire can also create inaccurate temperature measurements if insufficient pixel resolution is available across the wire diameter. Some postprocessing of the results may be necessary in this case. (Further details of these issues will be provided in a later article of this series.)

\section{ISOTHERMAL MECHANICAL RESPONSES}

We have used most of the above methods at various times, depending on the goals of the specific measurements. For now, however, our interest is focused on isothermal mechanical responses (over relevant temperature windows) of the same two commercial Nitinol wire alloys (Memry Corp., Bethel, CT) that were previously introduced in Part $1 .{ }^{1}$ As before, the alloy with stress-free austenite transformation temperatures above room temperature is termed "shape memory wire" (same material as used in Shaw ${ }^{8}$ and Shaw and Kyriakides ${ }^{9}$ ), while the alloy with transformations below room temperature is termed "superelastic wire" (same material as used in Chang et al. $\left.{ }^{10}\right)$.

\section{Experimental Setup}

The experiments to be presented on the SM and SE wires were performed in a liquid bath and an air chamber, respectively, as shown in Figs. 1 and 2. The custom-built liquid setup of Fig. 1 for SM wire experiments had a stainless steel specimen bath with a glass front and an o-ring seal in its base. This allowed the bath to be lowered for specimen installation into the grips and then raised, immersing the specimen and its instrumentation, for testing within an electromechanical testing machine. The long grip extension was made of stainless steel and was designed to be several orders of magnitude stiffer than the test specimens. The bath temperature was regulated by an external circulator (NESLAB RTE-110, Thermo Fisher Scientific, Waltham, MA) that reached temperatures in the range -20 to $120^{\circ} \mathrm{C}$, depending on the working fluid listed in Table 1. This range spanned the window of transformation temperatures for the SM wire. The fluid temperature was preset at the external circulator, accounting for any heat losses/gains along the fluid piping by measuring the specimen bath temperature by a temperature probe.

The load frame was operated under displacement control at the rather slow elongation rate of $\dot{\delta} / L= \pm 4 \times 10^{-4} s^{-1}$ to maintain nearly isothermal conditions. The nominal axial stress was measured as the ratio $\left(P / A_{0}\right)$ based on the measured tensile force $(P)$ and the original cross-sectional area $\left(A_{0}\right)$. Local strain measurement was obtained by a custombuilt, miniature, waterproof extensometer having a gage length between the knife-edges of $2.54 \mathrm{~mm}$ ( 0.1 inch) (see Shaw ${ }^{8}$ and Shaw and Kyriakides ${ }^{9}$ for details). The strain measured by the extensometer was the ratio $\left(\delta_{\mathrm{e}} / L_{\mathrm{e}}\right)$ of the elongation in the extensometer $\delta_{\mathrm{e}}$ to the gage length of the extensometer at zero load $L_{\mathrm{e}}$. Local temperature measurement was obtained by one or more small thermocouples attached to the specimen.

The SE wire required yet lower temperatures to span its transformation temperatures, so a commercial air chamber 


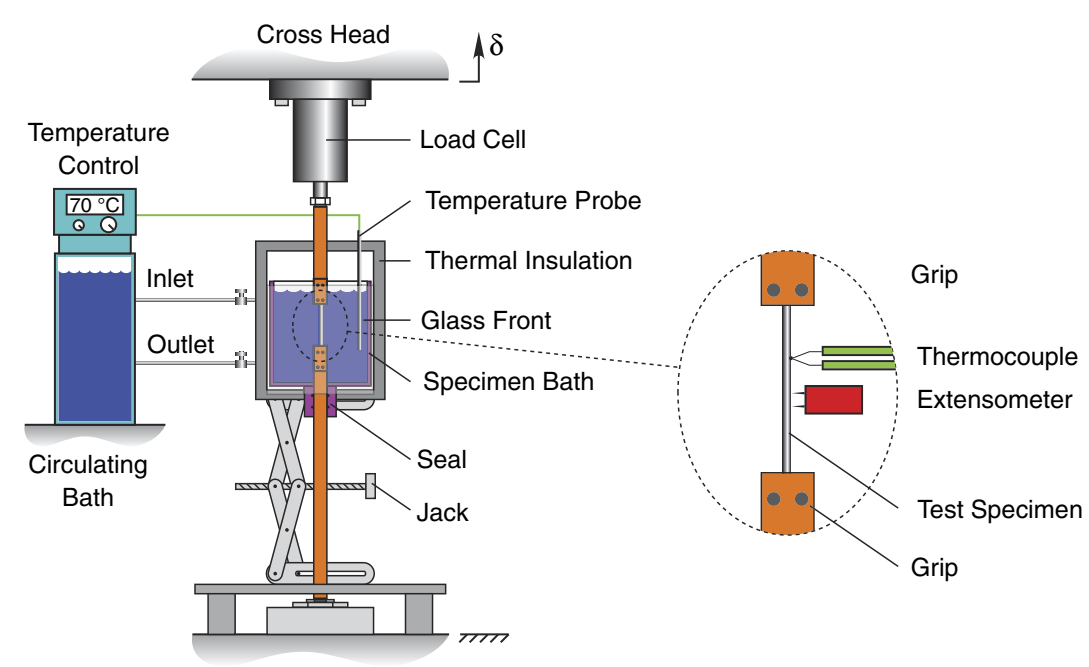

Fig. I: Setup for experiments on SM wire in a temperature-controlled bath

with liquid nitrogen cooling was used instead, as shown in Fig. 2. The top/bottom openings of the air chamber were sealed with thermal insulation to be as airtight as possible without constraining the motion of the crosshead. Strain was measured through the front observation window by a laser extensometer, with a gage length near $60 \mathrm{~mm}$. Because air is more thermally insulating than liquid, the strain rate was reduced to a very slow $\dot{\delta} / L= \pm 5 \times 10^{-5} s^{-1}$ to avoid selfheating/cooling during phase transformation.

In both cases, the axial load on the specimen was measured by a high-quality $5-\mathrm{kN}$ (1000 lb) load cell that was balanced to account for the weight of fixturing above the specimen. The load cell was shielded on the underside to protect it from overheating or condensation/frost buildup due to convection and conduction from within the chamber. A small fan kept room temperature air blowing across it to minimize temperature changes in the load cell that would otherwise result in load measurement drift.
For all experiments presented, the uncertainty of the measured load and strain is on the order of the thickness of the thickest line in the figure. For data derived from construction lines or estimated from response curves, the uncertainty is about the size of the diameter of circles shown in the plot, with a worst-case error of about three circle diameters in the few cases where points were estimated by "eye" (explicit error bars are omitted for clarity).

\section{Specimen Materials}

Reviewing information gathered from the DSC thermograms in Part 1, Table 2 shows stress-free transformation temperatures between the three phases: austenite $(A)$, thermal- $R$ phase $(R)$, and thermal-martensite $(M)$. Specific heat and latent heat of transformation values for the three transformations $M \rightarrow A\left(\Lambda_{\mathrm{MA}}\right), A \rightarrow R\left(\Lambda_{\mathrm{AR}}\right)$, and $R \rightarrow M\left(\Lambda_{\mathrm{RM}}\right)$ are provided in Table 3. The SM wire had a diameter of $1.016 \mathrm{~mm}(0.040$ inch), while the SE wire had a diameter of $0.762 \mathrm{~mm}(0.030$

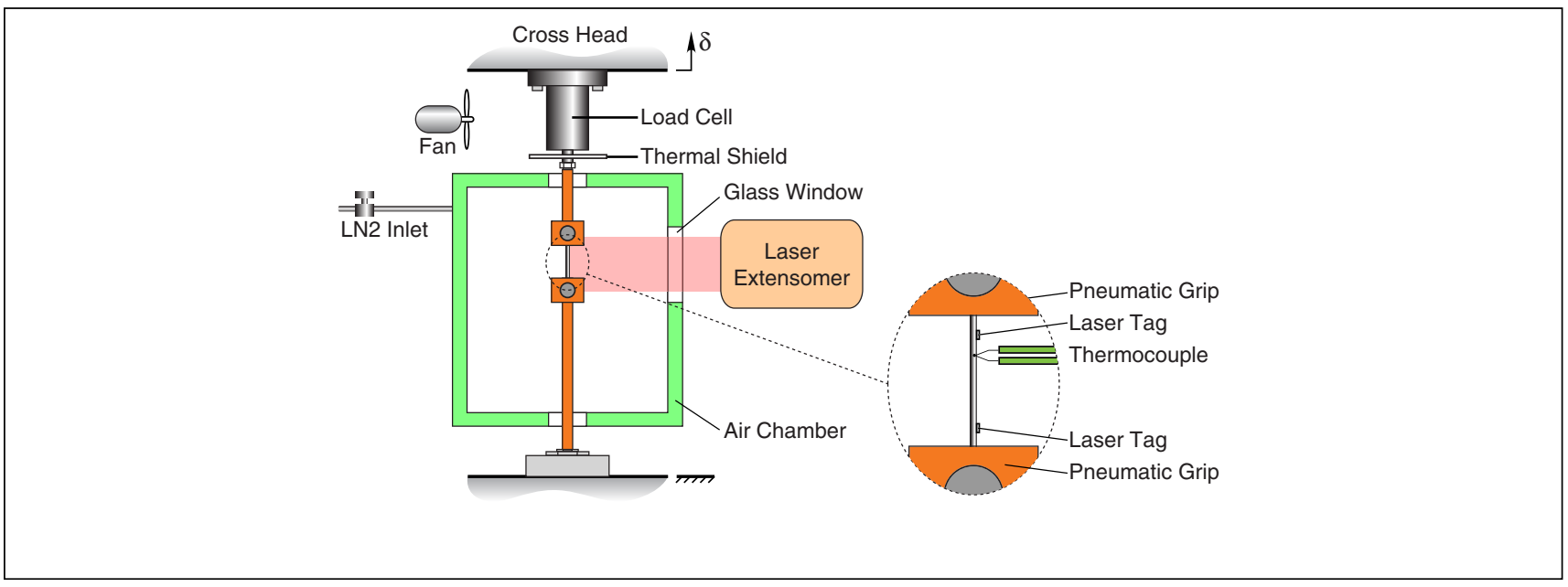

Fig. 2: Setup for experiments on SE wire in an air chamber 
Table I-Working fluid for SM wire experiments

\begin{tabular}{ll}
\hline WORKING FLUID & TEMPERATURE RANGE $\left({ }^{\circ} \mathbf{C}\right)$ \\
\hline Glycol/water & -18 to 10 \\
Water & 10 to 80 \\
Mineral oil & 80 to 100 \\
\hline
\end{tabular}

inch). The wire length between the grips $(L)$ was about 69 and $127 \mathrm{~mm}$ for the respective alloys, yet each was measured accurately (by gage blocks and small crosshead motion after specimen installation) at the start of each experiment at a very small tensile load, just enough to remove any slack in the wire. All experiments shown below were performed on specimens from the same spool of wire, and each experiment was performed on a different wire specimen to avoid introducing any unwanted history effects.

\section{SM Wire Responses}

A series of isothermal mechanical responses of the SM wire, one every $10^{\circ} \mathrm{C}$, is shown in Fig. 3. The material was first tested in its "as-received" state, so below $0^{\circ} \mathrm{C}$, the initial material phase was a mixture of thermal- $R$ phase $(R)$ and thermal-martensite $(M)$. At these low temperatures, the response during loading is soft and nonlinear. The initial knee in the stress-strain curve before $1 \%$ strain is due to reorientation of thermal- $R$ to tensile- $R\left(R^{+}\right)$. Subsequent to this initial knee, the response briefly stiffens but then flattens into a stress plateau as the material is transformed to $M^{+}$. The initial load-unload cycle was taken to a strain just beyond the end of the stress plateau, near 4-5\%, leaving a residual strain upon unloading that could potentially be recovered (not shown) upon stress-free heating by the SM effect. For temperatures below and including $40^{\circ} \mathrm{C}$, a second load-unload cycle was taken to a maximum strain of about $7 \%$ before unloading, showing the postplateau response of $M^{+}$. As the temperature was raised in the sequence of experiments in Fig. 3, one sees the loading plateaus $(\dot{\delta}>0)$ trend upward in stress and the unloading responses $(\delta<0)$ becoming more nonlinear. Note that above $20^{\circ} \mathrm{C}$, the loading responses develop distinct stress plateaus, and these occur at progressively higher stresses as the temperature of the experiment is raised. A dramatic change in the unloading response occurs as the temperature reaches $40^{\circ} \mathrm{C}$, in which the residual strain is less than $1 \%$ after the first load-unload cycle. This temperature happens to be near the average of $A_{\mathrm{s}}$ and $A_{\mathrm{f}}$ (Table 2).

At higher temperatures, $50^{\circ} \mathrm{C}$ and above, the responses are SE. These consist of an initially stiff loading response associated with elastic loading of $A$, followed by a sudden tangent

Table 2-Transformation temperatures as measured by DSC, with uncertainty of $\pm 2^{\circ} \mathrm{C}^{\prime}$

\begin{tabular}{lcccccc}
\hline ALLOY & $\boldsymbol{M}_{\mathrm{f}}\left({ }^{\circ} \mathrm{C}\right)$ & $\boldsymbol{M}_{\mathrm{s}}\left({ }^{\circ} \mathbf{C}\right)$ & $\boldsymbol{R}_{\mathrm{f}}\left({ }^{\circ} \mathrm{C}\right)$ & $\boldsymbol{R}_{\mathrm{s}}\left({ }^{\circ} \mathrm{C}\right)$ & $\boldsymbol{A}_{\mathrm{s}}\left({ }^{\circ} \mathrm{C}\right)$ & $\boldsymbol{A}_{\mathrm{f}}\left({ }^{\circ} \mathbf{C}\right)$ \\
\hline SM wire & $<-50$ & -7 & 31 & 52 & 26 & 56 \\
SE wire & -120 & -73 & -30 & 13 & -30 & 13 \\
\hline
\end{tabular}

Table 3-Specific heats $\left(c_{\mathrm{o}} \pm 0.02 \mathrm{~J} /(\mathrm{gK})\right)$ and latent heats of transformation $(\Lambda \pm \mathrm{J} / \mathrm{g})$ as measured by DSC

\begin{tabular}{lcccc}
\hline ALLOY & $c_{\mathrm{o}}(\mathrm{J} /(\mathrm{gK}))$ & $\Lambda_{\mathrm{MA}}(\mathrm{J} / \mathrm{g})$ & $\Lambda_{\mathrm{AR}}(\mathrm{J} / \mathrm{g})$ & $\Lambda_{\mathrm{RM}}(\mathrm{J} / \mathrm{g})$ \\
\hline SM wire & 0.45 & 19.7 & -6.7 & $\approx-7$ \\
SE wire & 0.45 & 15 & -5.0 & $\approx-6$ \\
\hline
\end{tabular}

modulus change to 0 , during which $A \rightarrow M^{+}$transformation occurs along a stress plateau. A subsequent sharp upturn in the stress response occurs at the end of the plateau (see, e.g., the $70^{\circ} \mathrm{C}$ response), and elongation rate was reversed just beyond this point. Upon subsequent unloading, a nonlinear path is taken initially, but then, another critical stress is reached where the elongation decreases along another stress plateau, during which the reverse $M^{+} \rightarrow A$ transformation occurs. Once the elongation intersects the original austenite loading path, the stress takes a sharp downturn and unloading finishes along the austenite elastic curve. For all the SE responses shown, unloading commenced just after exhaustion of the loading plateau to avoid introducing additional plasticity effects that would further complicate the interpretation of the response. Even so, one can see that perfect superelasticity no longer occurs for responses at $80^{\circ} \mathrm{C}$ and above, where a progressively larger residual strain remains after unloading as the temperature is increased. In fact, the extent of the unloading stress plateau has nearly vanished at $100^{\circ} \mathrm{C}$.

For conventional metals, the critical stress at the onset of significant nonlinearity would be thought of as a plastic yield point. Increasing the temperature normally softens the yield point of a conventional metal (although the effect is rather gradual until the temperature gets quite high). By contrast, the apparent "yield point" of an SMA increases dramatically

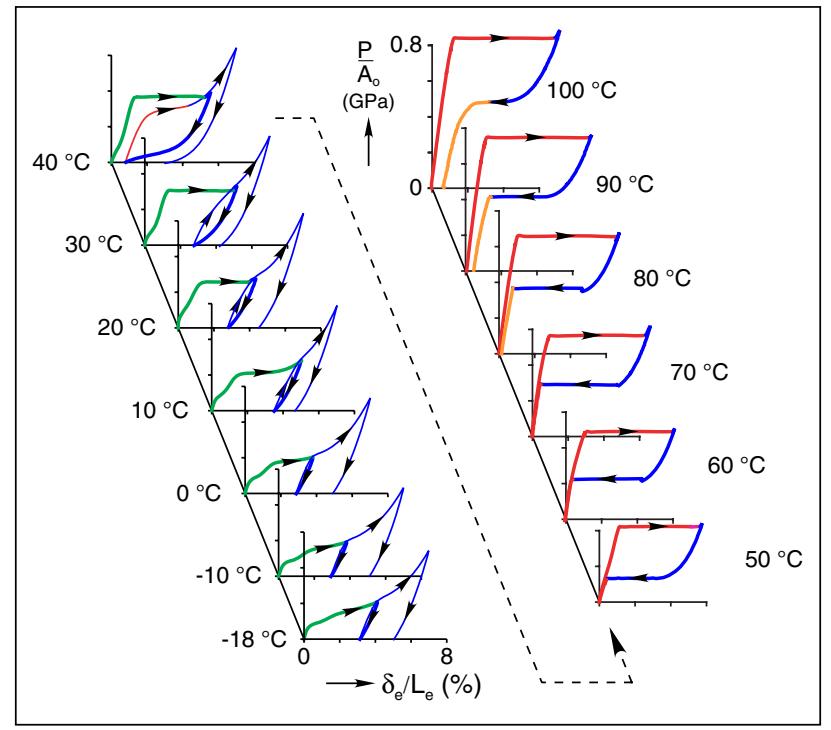

Fig. 3: Isothermal mechanical responses of SM wire at selected temperatures. Data taken from Shaw ${ }^{8}$ and Shaw and Kyriakides ${ }^{9}$ 


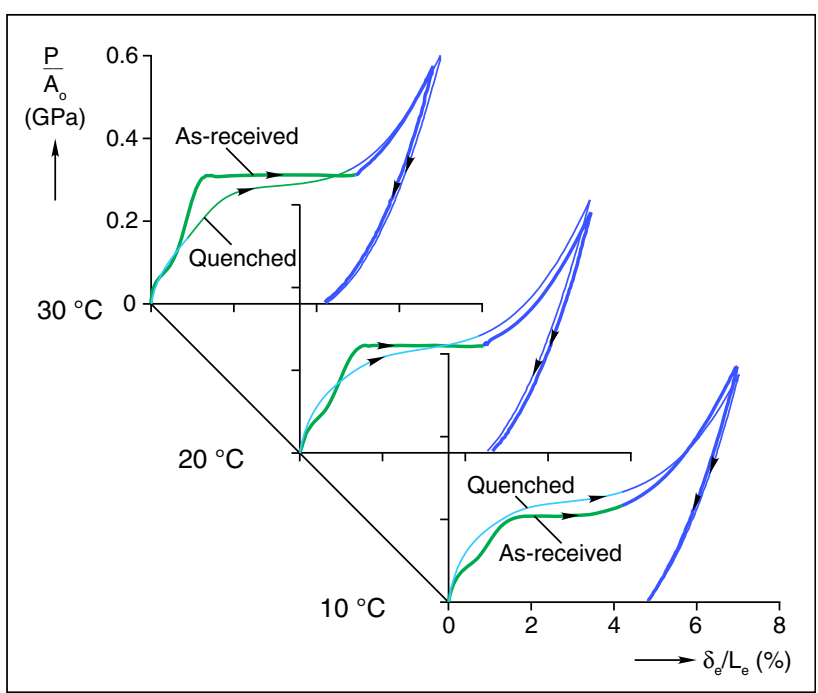

Fig. 4: Comparison of liquid nitrogen-quenched material (thin line) and as-received material (thick line) responses of SM wire at intermediate temperatures. Data taken from Shaw and Kyriakides ${ }^{9}$

as the temperature is raised. According to this view, the SMA material seems to get significantly stronger as the temperature is raised, contrary to conventional materials, which tends to catch the SMA novice by surprise. The underlying reason is that it is not a yield point associated with actual plasticity but rather a transformation stress where the parent phase becomes unstable in favor of the daughter phase. As the temperature rises away from the cold temperatures where $M^{+}$is naturally stable (stress free), it requires progressively larger stresses to destabilize $A$, that is, to stress induce $M^{+}$. Furthermore, if the temperature continues to rise, the transformation stress will eventually exceed that which causes actual plastic slip.

Notice again that for temperatures between 50 and $70^{\circ} \mathrm{C}$, perfect superelasticity occurs, but a small and then progressively larger amount of residual strain exists upon unloading at higher temperatures. This is caused by some amount of plastic deformation accompanying $A \rightarrow M^{+}$transformation for transformation stresses between 650 and $850 \mathrm{MPa}$ in the experiments at $80-100^{\circ} \mathrm{C}$. Although not shown, stress-free heating can recover some of this residual strain, but not all, indicating actual unrecoverable plasticity has occurred. This is not surprising, considering the stress reached high values during loading, where the required transformation stress has exceeded the true yield stress of the material. As the temperature increases further (not shown), progressively smaller portions of the residual strain at the end of unloading would be recovered by further stress-free heating. At excessively high temperatures, the unloading plateau vanishes altogether (becoming just nonlinear, akin to the $100^{\circ} \mathrm{C}$ unloading response shown) and the permanent strain grows dramatically. Consequently, there is a higher temperature (well above $100^{\circ} \mathrm{C}$ ), called $A_{\mathrm{d}}$, above which little stress-induced martensite is recovered upon unloading, and the material has a mechanical response similar to that of a conventional, ductile elastoplastic material.

As indicated by DSC, the stress-free phase of the material is not uniquely identified at certain intermediate temperatures. For example, Fig. 4 shows three experiments between 10 and $30^{\circ} \mathrm{C}$ that each compares mechanical responses for as-received specimens and specimens that were quenched in liquid nitrogen $\left(-196^{\circ} \mathrm{C}\right)$ before the start of the experiment. The asreceived specimens were predominately thermal- $R$ when the experiment was started (since they presumably were cooled to room temperature after the wire was processed at much high temperatures), whereas the quenched specimens were predominately thermal- $M$ after being allowed to warm to their test temperature from $-196^{\circ} \mathrm{C}$. A double knee is apparent in the first $1.5 \%$ strain of the responses for the initially thermal$R$ material (as-received) as it undergoes a multistep transformation $R \rightarrow R^{+} \rightarrow M^{+}$, first reorienting thermal- $R$ to tensile- $R$ and then transforming to tensile- $M$. The quenched material, on the other hand, exhibits a single knee associated with reorientation of martensite $M \rightarrow M^{+}$.

The different responses, even for experiments under identical thermomechanical conditions, again underscore the importance of knowing the prior material history before interpreting them. Nevertheless, once the specimen is taken beyond about $4 \%$ strain, the phase is largely $M^{+}$, and subsequent loading and unloading responses are quite similar between the as-received and the quenched materials. Although not
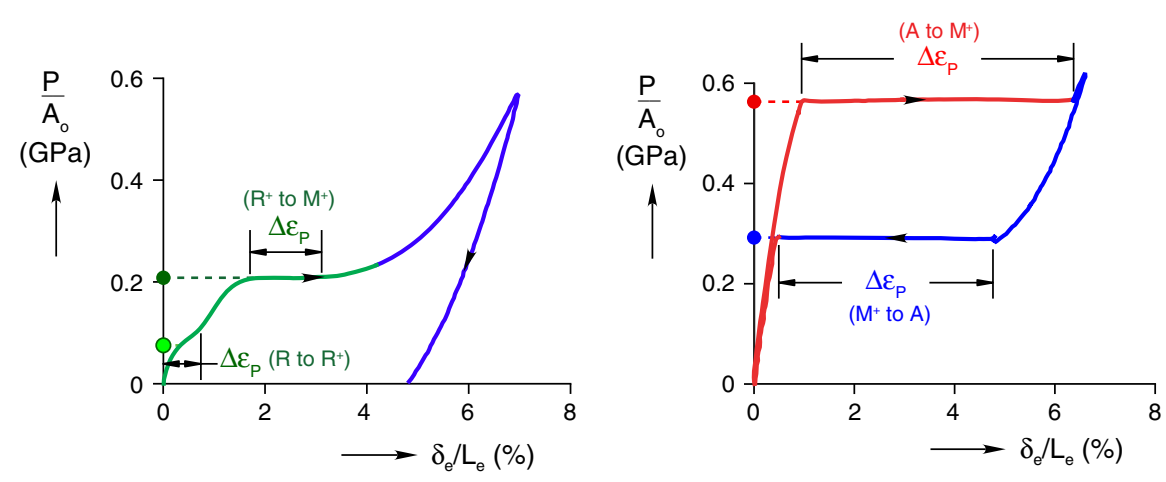

Fig. 5: Example of mechanical responses showing how characteristic plateau stresses and strains are extracted: (a) $10^{\circ} \mathrm{C}$ experiment and (b) $70^{\circ} \mathrm{C}$ experiment 


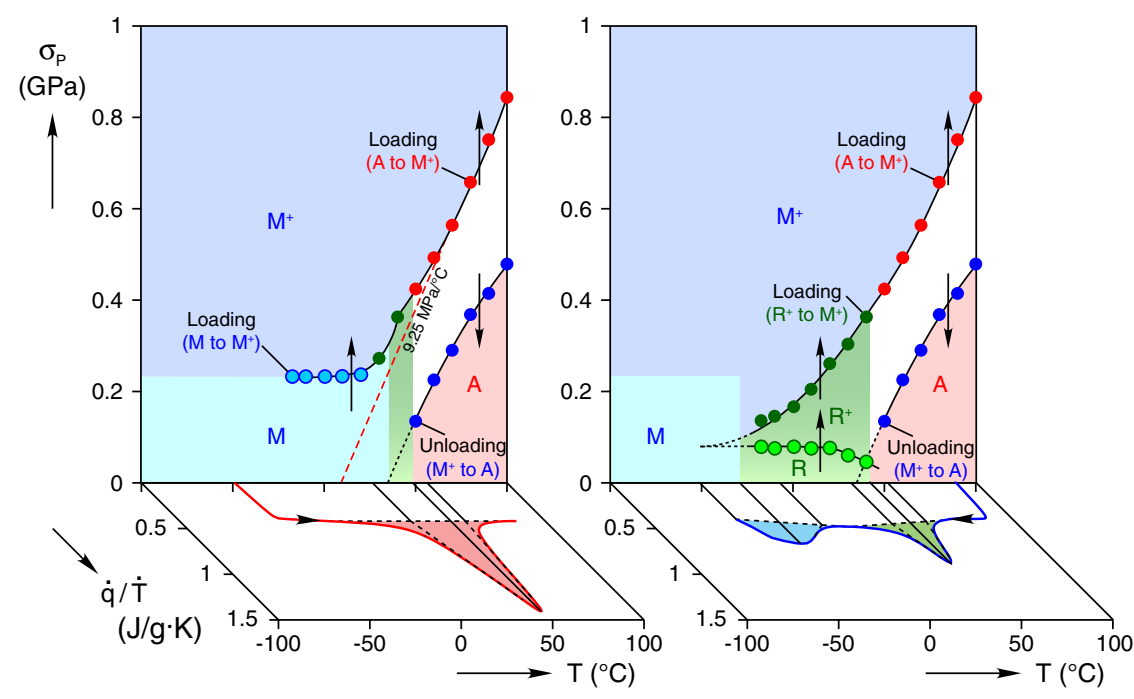

Fig. 6: Characteristic transformation stresses of SM wire: initially quenched in liquid nitrogen (left) and as-received (right). Respective heating and cooling DSC thermograms also shown along the stress-free axis for reference

shown in Fig. 4, additional experiments were performed on quenched wire over the entire temperature range considered, but little change in the response was observed at temperatures above $40^{\circ} \mathrm{C}$ from the as-received responses shown in Fig. 3.

A useful map of the material behavior is obtained by plotting the stress of the plateaus and their extents in strain as a function of temperature. Figure 5 depicts how plateau stresses $\left(\sigma_{\mathrm{P}}\right)$ and strain jumps $\left(\Delta \varepsilon_{\mathrm{P}}\right)$ are extracted from the curves in Fig. 4. Characteristic stresses are found from either a visual estimate of the knee of the curve or wherever distinct stress plateaus occur. Transformation strains are likewise obtained from the associated strain change that occurs during changes in microstructure (reorientation) or phase (length of stress plateaus). Strictly speaking, phase transformation does not occur exclusively during stress plateaus, that is, some microstructural changes occur just before and after the plateaus and are responsible for most of the nonlinearity observed at

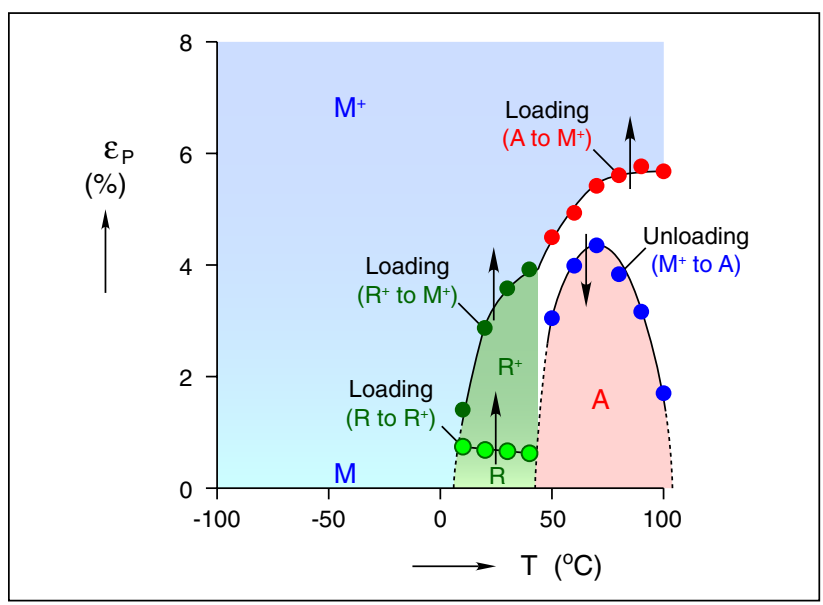

Fig. 7: Characteristic transformation strains of SM wire (asreceived) either ends of the plateau. However, the strain change due to strict elasticity versus transformation in these portions of the response is difficult to distinguish precisely by macroscopic measurements, so only the transformation strain across the stress plateau will be reported.

Figure 6 plots characteristic stresses versus temperature for the SM wire starting in the as-received and quenched states. The DSC heating and cooling thermograms are shown along the stress-free axis for reference. While these transformations are hysteretic, the plot can still be interpreted as a quasiphase diagram in temperature-stress space. The plot on the left shows quenched wire that starts as $M$ at temperatures below about $0^{\circ} \mathrm{C}$, while the right plot shows as-received wire starting as $R$ at similarly low temperatures. The shaded areas

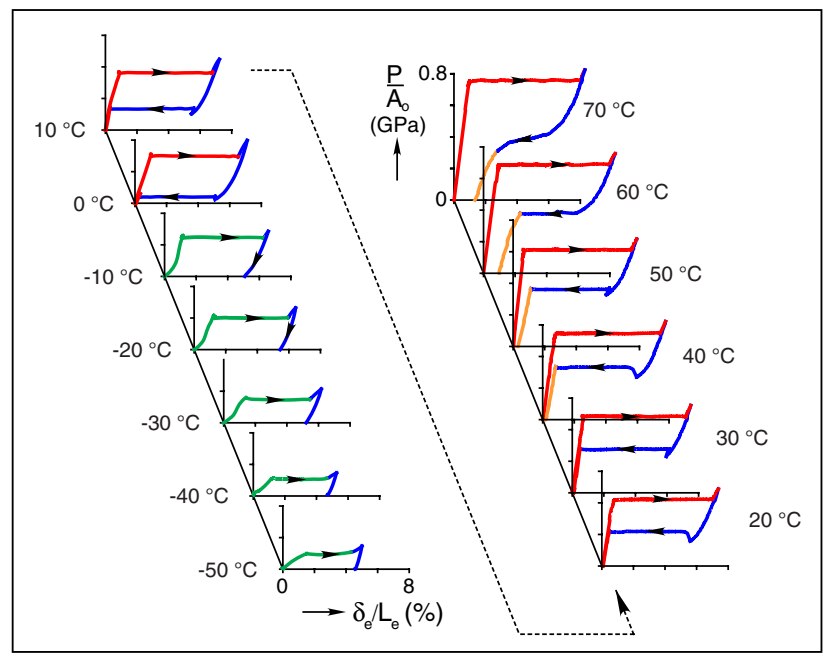

Fig. 8: Isothermal mechanical responses of as-received SE wire at selected temperatures. Data taken from ladicola ${ }^{\prime \prime}$ and Chang et al. ${ }^{10}$ 


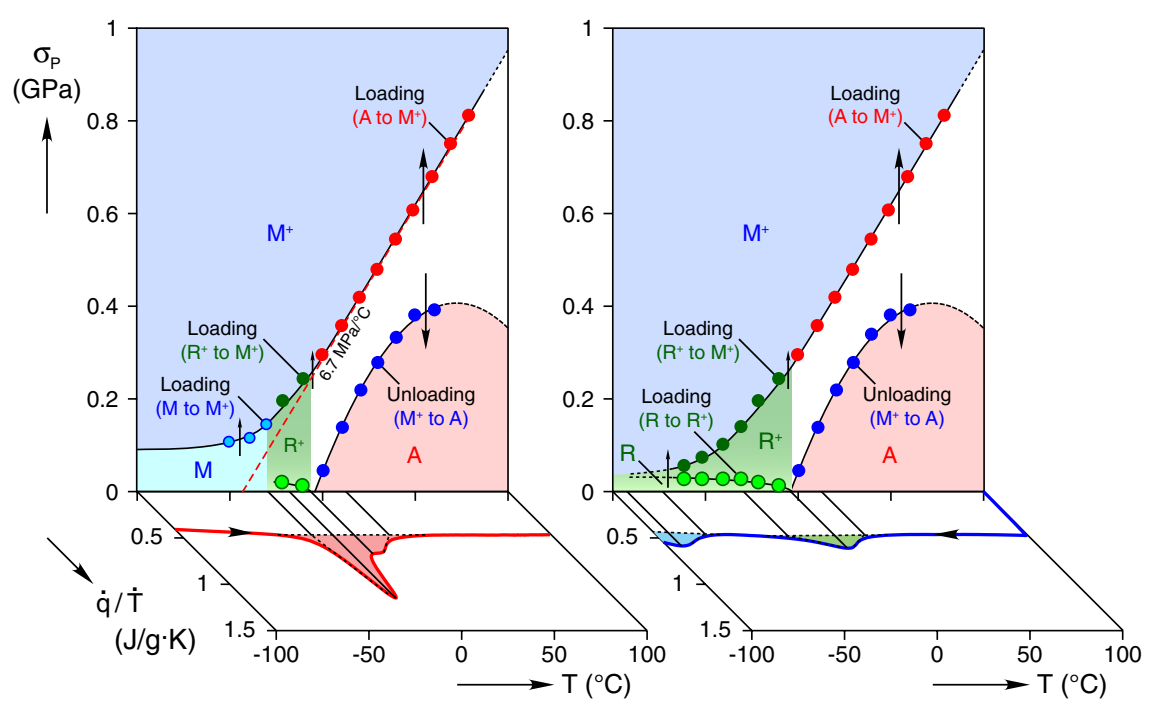

Fig. 9: Characteristic transformation stresses of SE wire: initially quenched in liquid nitrogen (left) and as-received (right). Respective heating and cooling DSC thermograms also shown along the stress-free axis for reference

depict the stable phase. In areas between the lines, the phase is dependent on loading history, so the vertical arrows show the direction that a phase boundary is crossed. Quenching has little to no effect in the high temperature SE range but does have an effect at intermediate to low temperatures. The plots also indicate that reorientation of $R \rightarrow R^{+}$or $M \rightarrow M^{+}$is relatively independent of temperature, at least for the temperature range shown. The transformation stresses for $R^{+} \rightarrow M^{+}$ and $A \rightarrow M^{+}$, however, are extremely temperature dependent, rising from about 150 to $850 \mathrm{MPa}$ (as-received material), nearly a factor of 6 , over the $120^{\circ} \mathrm{C}$ temperature range of the data shown. The slope for $A \rightarrow M^{+}$transformation is about 9.25 $\mathrm{MPa} /{ }^{\circ} \mathrm{C}$ at the high end of the temperature range, for both quenched and as-received wires.

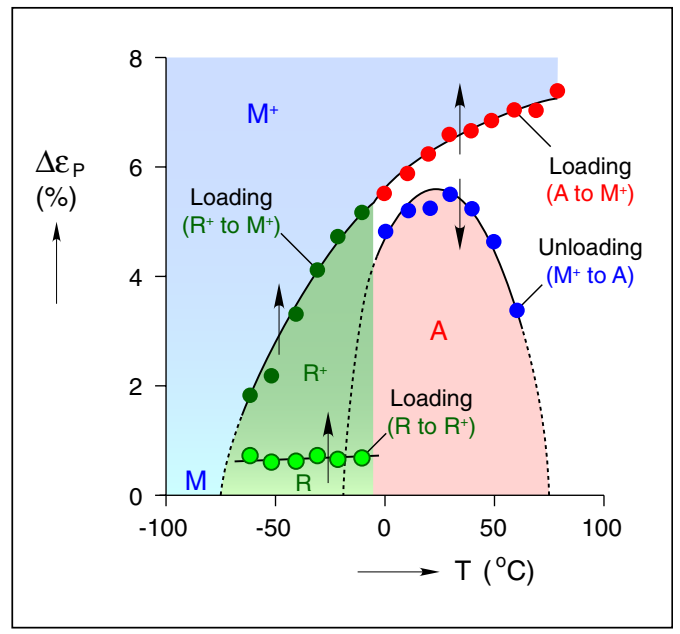

Fig. 10: Characteristic transformation strains of SE wire (as-received)
A summary of transformation strains are presented in a similar manner in Fig. 7. The trend for each type of transformation is quite nonlinear, tracing a quadratic-like curve in each case. The $R \rightarrow R^{+}$strain is the exception, once again being relatively insensitive to temperature (although these were estimated by eye from the response curves). Both $M^{+} \rightarrow A$ and $A \rightarrow M^{+}$strains increase with temperature until they reach maxima at 70 and $90^{\circ} \mathrm{C}$, respectively. At the highest temperature $\left(100^{\circ} \mathrm{C}\right)$, increasing plasticity causes the $M^{+} \rightarrow A$ plateau strain to fall below $2 \%$, while $A \rightarrow M^{+}$just begins to decrease from its maximum of about $5.8 \%$ strain.

\section{SE Wire Responses}

For comparison, the same type of data presented above is now provided for the SE wire. Isothermal mechanical responses of the as-received SE wire are shown in Fig. 8, but the experiments were performed in an air chamber over a temperature range of -50 to $70^{\circ} \mathrm{C}$. The responses have similar trends to that of the SM wire of Fig. 3 but shifted to lower temperatures. Similar to the SM wire, the lowest temperature responses of the SE wire have low plateau stresses and a residual strain upon unloading. Superelasticity is exhibited in the responses above $0^{\circ} \mathrm{C}$, but imperfect superelasticity with a residual strain after unloading occurs in responses at $40^{\circ} \mathrm{C}$ and above. Plots of transformation strains and stresses are shown in Figs. 9 and 10, respectively (including data from a few experiments not shown in Fig. 8), which bring out a few quantitative differences with the SM wire. Most noticeable is the lower $M \rightarrow M^{+}$transformation stress for the SE wire, less than half that of the SM wire in Fig. 6. The slope of the $A \rightarrow M^{+}$transformation for the SE wire is about 6.7 $\mathrm{MPa} /{ }^{\circ} \mathrm{C}$, as shown in Fig. 9, less than the corresponding $9.25 \mathrm{MPa} /{ }^{\circ} \mathrm{C}$ of the $\mathrm{SM}$ wire. Figure 10 shows the SE wire $A \rightarrow M^{+}$transformation strain continuing to rise in the temperature range tested, while the corresponding transformation strain of the SM wire reaches a peak value and begins decreasing at the highest temperature in Fig. 7. No data 
(a)

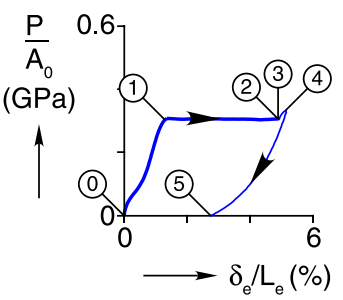

(b)

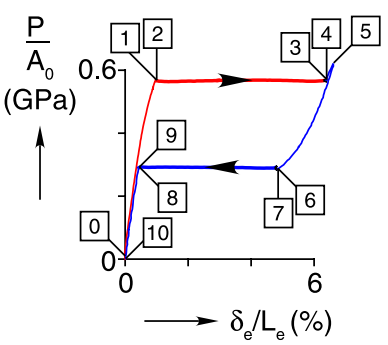

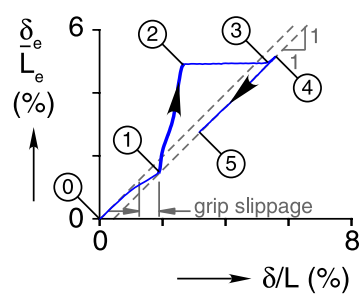

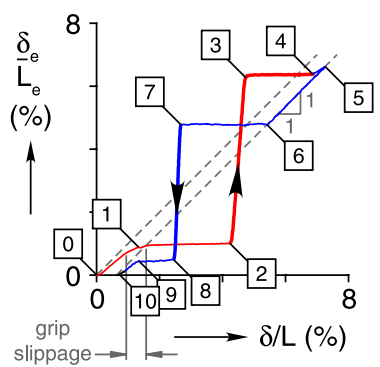

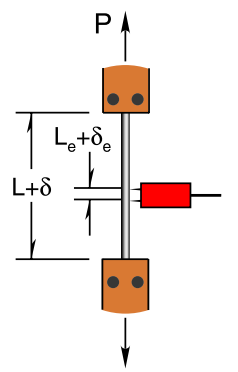

Fig. II: Axial stress versus local strain responses (left) and measured local versus global strain responses (right) in SM wire during two experiments: (a) $30^{\circ} \mathrm{C}$ and (b) $70^{\circ} \mathrm{C}$

points are given for the $M^{+} \rightarrow A$ transformation for temperatures above $60^{\circ} \mathrm{C}$ in Figs. 9 and 10 since the unloading plateau (Fig. 8) was too nonlinear to extract distinct values for stress or transformation strain. Also, the maximum plateau strain measured in the SE wire is about $7.4 \%$, significantly larger than the SM wire maximum value of about $5.8 \%$.

\section{MATERIAL INSTABILITY AND TRANSFORMATION FRONTS}

Difficulties in performing experiments on SMAs are often rooted in one (or a combination) of two phenomena. The first is material-level instabilities, and the second is thermomechanical coupling associated with the latent heat of transfor- mation. Both create particular, and somewhat subtle, issues for the SMA experimentalist. Here, we discuss the experimental issues associated with the first one and leave the second one for the next article.

During uniaxial stress-induced transformation, many NiTi alloys have (now) well-known material-level instabilities ${ }^{2,9}$ that give rise to localized deformation and propagating transformation fronts. As an example, Fig. 11 shows detailed measurements at two different temperatures $\left(70\right.$ and $\left.30^{\circ} \mathrm{C}\right)$ for the $\mathrm{SM}$ wire. On the left, the mechanical responses are shown with axial strain measured by a miniature mechanical extensometer $\left(\delta_{\mathrm{e}} / L_{\mathrm{e}}\right)$, and on the right, the extensometer response (local strain) is compared to the grip motion, normalized as $\delta / L$ (global strain). During testing of conventional stable

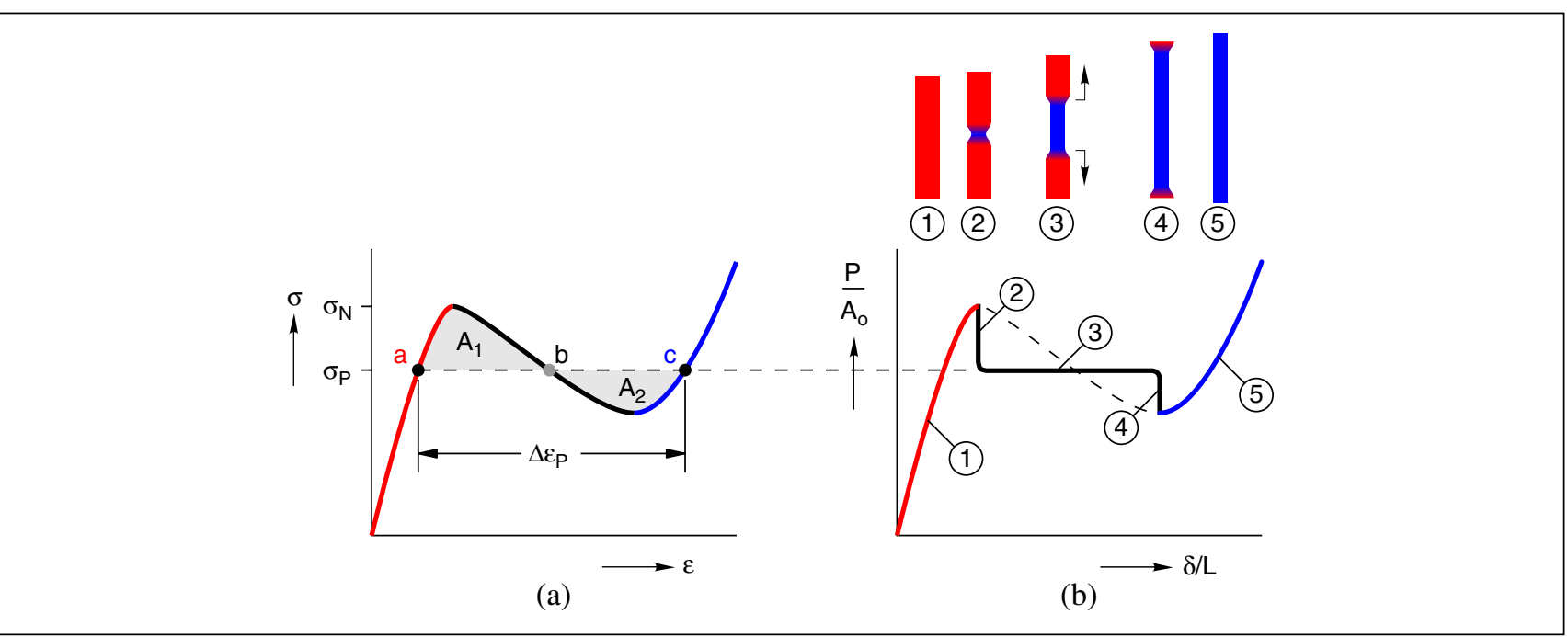

Fig. 12: Schematics of (a) local stress-strain response and (b) global force-elongation ("structural") response with specimen configurations before, during, and after $A \rightarrow M^{+}$transformation 
materials, one would expect the two strain measurements (local and global) to agree, at least approximately, and simply trace a $45^{\circ}$ line in the plot (dashed line). Focusing first on the $30^{\circ} \mathrm{C}$ response in Fig. 11a, this is what occurs initially during loading between states 0 and 1 during $R \rightarrow R^{+}$. During the stress plateau between (1) and (2) $\left(R^{+} \rightarrow M^{+}\right)$, however, the extensometer records a more rapid increase in strain $\left(\delta_{\mathrm{e}} / L_{\mathrm{e}}\right)$ compared to the global strain $(\delta / L)$, but then between (2) and (3), the local strain stays static while the global strain increases (and the point does not move in the force vs. local strain response). Loading continues briefly between (3) and (4), where an upturn in the force response is measured, and the local versus global strain traces again along a $45^{\circ}$ (but slightly shifted) line. At (4), the direction of the crosshead is reversed and the specimen unloads along a nonlinear path in the force response, and the local versus global strain traces backward along the $45^{\circ}$ line until the force is 0 at point (5). One also notices that the local strain diverges somewhat from the global strain near point (1), but this is an artifact of the experiment where some grip slippage has occurred (hence, the offset between the two $45^{\circ}$ dashed lines). This is caused by stress concentrations and localized axial straining (and thus lateral thinning of the wire) at the grips, creating a small amount of "inch-worming" of the wire out of the grips (about $0.5 \%$ global strain) that is difficult to avoid in this setup. Thus, a global force-elongation response (not shown) would erroneously show a larger permanent strain than actually occurs in the material.

The stress plateau during $R^{+} \rightarrow M^{+}$has a zero tangent modulus and is associated with local/global strain responses that diverge at the start of transformation (1) and then reconverge at the end of transformation (3. In this case, transformation first localizes within the gage section of the extensometer (and a rapid increase in local strain is measured) and then deformation progresses outside the extensometer's gage section (and no change is recorded by the extensometer) while overall elongation continues until the specimen has been completely consumed by the new phase $\left(M^{+}\right)$. Conversely, one observes that wherever the force-elongation response maintains a positive tangent modulus, the local and global strain measurements follow a $45^{\circ}$ line, meaning the whole specimen undergoes uniform deformation. Accordingly, reorienting of $R \rightarrow R^{+}$during loading and retwinning of $M^{+}$during unloading (responsible for the nonlinear path) are stable processes in this alloy.

Figure 11b shows similar phenomena in the SE response of the SM wire at $70^{\circ} \mathrm{C}$, but now, localization occurs during both loading $\left(\mathrm{A} \rightarrow M^{+}\right.$between 1 and 4$)$ and unloading $\left(M^{+} \rightarrow A\right.$ between 6 and 9 ). During both transformations, the local strain first stays static, then jumps, and then stays static again. This is caused by transformation occurring by the propagation of one or more boundaries separating nearly uniform high-strain and low-strain regions. The axial extent of such a transformation front is only a few wire radii, where a steep gradient in strain exists; thus, it can be considered a propagating neck in the specimen. The extensometer, therefore, will only record a change in strain when a transformation front passes through its measurement region. The specific times at which the strain jumps are recorded depending on the location of the extensometer and the particular motion of transformation fronts.
Such localization and propagation phenomena, while uncommon in materials testing, certainly do exist in other materials but for different microscopic reasons. Examples include mild steel that exhibits Lüders bands during uniaxial testing ${ }^{11-13}$ and high-density polyethylene ${ }^{14,15}$ that exhibits large strain propagating necks during tensile cold drawing. Figure 12 shows a schematic of the common underlying cause of these phenomena. The material constitutive law (local stress-strain) has an up-down-up character (Fig. 12a), which gives rise to the force-elongation, or "structural," response (Fig. 12b) under displacement control, consisting of the following sequence: (1), an initial rising curve; (2), a nucleation peak initiated at $\sigma_{\mathrm{N}}$ with a drop in load to $\sigma_{\mathrm{P}}$ at constant elongation; 3, a force plateau at $\sigma_{\mathrm{P}}$ with increasing elongation and propagating fronts; (4, another drop in load again at constant elongation as the strain delocalizes; and (5), a rising curve after the plateau elongation has been exhausted and specimen has been completely converted to the high-strain phase. The negative slope in the local constitutive law represents an unstable segment that is energetically unfavorable unless the material is otherwise constrained, as in the neck region by material on either side. At the onset of instability, a finite portion of the wire transforms in a dynamic manner 2), creating a high-strain inclusion somewhere in the wire length. Since the total elongation is being controlled, that is, fixed during this instant, the remaining material must unload, so the load drops. During subsequent propagation of fronts away from the nucleation site, equilibrium requires that the force be constant along the length, so the material can exist in states (a), (b), or (c), as shown in the figure, but only states (a) and (c) are stable. As such, the unstable portion of the constitutive law is difficult to measure in practice and must be inferred from the global structural response as follows. The plateau stress $\left(\sigma_{\mathrm{P}}\right)$ and plateau strain $\left(\Delta \varepsilon_{\mathrm{P}}\right)$ can be found by the construction shown in the figure where the shaded areas are equal $\left(\mathrm{A}_{1}=\mathrm{A}_{2}\right)$. The theory is based on nonlinear elasticity ${ }^{16}$ but gives a reasonable approximation even when dissipation/hysteresis is involved. In the hysteretic case, one imagines two underlying local stressstrain curves, an upper one for loading $(\dot{\delta}>0)$ and a lower one for unloading $(\dot{\delta}<0)$, each having an up-down-up character, thereby giving rise to two stress plateaus. ${ }^{10}$

Nucleation peaks, however, are absent in the experiments of Fig. 11 at the onset of transformation during loading. Unless the experiment is carefully designed, stress concentrations at the grips will usually suppress a portion of, or the entire, load peak that accompanies the nucleation of an $M^{+}$region, thereby giving the appearance of an up-flat-up response during loading. The measured nucleation stress $\sigma_{\mathrm{N}}$ is imperfection sensitive, that is, will be reduced by geometric or loading imperfections from a theoretical perfect upper bound. In our case, the multiaxial stress concentrations at the grip entrances act as starters for $R^{+} \rightarrow M^{+}$or $A \rightarrow M^{+}$transformation and are large enough to effectively mask the nucleation stress peak. If one looks closely, however, at the unloading responses at $60-80^{\circ} \mathrm{C}$ in Fig. 3 and the unloading responses between 0 and $50^{\circ} \mathrm{C}$ in Fig. 8, nucleation peaks can be seen at the onset of the $M^{+} \rightarrow A$ transformation plateaus. The nucleation peaks upon unloading are upside down, similar to state (4) of Fig. 12, since the strain is decreasing from a large value. These unloading peaks are apparent since the onset of $M^{+} \rightarrow A$ transformation began somewhere away from the grips since stress concentrations do not favor 
this reverse transformation. (A later article in this series will show how to better uncover all these nucleation peaks, during loading and unloading, and they will be shown to play an important role in the evolution of transformation, especially at higher loading rates.)

Interestingly, analogous localization and propagation phenomena also exist in certain structural problems, including, for example, crushing of honeycombs and foams with localized densification bands, ${ }^{17,18}$ external pressure of long pipes with propagating buckles, ${ }^{19}$ and inflation of rubber tubes with propagating bulges. ${ }^{20}$ The common feature is that the local structural response becomes unstable but then stiffens at larger strains due to material stiffening or internal contact mechanisms, that is, the "local" response (cell or cross-section) has an up-down-up character. ${ }^{21}$ In these cases, material points are locally stable, but instability occurs by geometric softening. By contrast, SMA wire instabilities are the result of both geometric effects (wire thinning) and local material instability (phase transformation at the microscale).

\section{SUMMARY AND CONCLUSIONS}

Our intent in this continuing series of articles is to provide recommendations for characterizing Nitinol SMA wire. We hope to forewarn experimentalists who are relatively inexperienced with SMAs of some pitfalls regarding experimental technique and interpretation of data.

In this article, Part 2, we outlined the task of isothermal mechanical characterization of SMA wire. This started with a discussion of experimental setups, from the loading method to measurement techniques to temperature control, covering multiple techniques for each goal, ranging from simple to complex. Next, we presented a series of isothermal experiments for both SM and SE SMA wire. Transformation stresses and strains were extracted from the experiments and plotted against both temperature and DSC data. The plots showed the martensite reorientation stress for SM and SE wire to be about 240 and $100 \mathrm{MPa}$ and the slope of the $A \rightarrow M^{+}$interfaces to be 9.25 and $6.6 \mathrm{MPa} /{ }^{\circ} \mathrm{C}$, respectively. We also showed what effect the starting material phase, $A, M$, or $R$, can have on the mechanical response. Finally, we discussed how to address experimental difficulties and interpretation associated with material instability and propagating transformation fronts. The forthcoming article in this series, Part 3, will treat loading rate effects and the influence of thermomechanical coupling.

\section{ACKNOWLEDGMENTS}

We thank Nilesh Mankame (General Motors) for the invitation to write this series of articles. We gratefully acknowledge the financial support for this work provided by the National Science Foundation and General Motors Corp. through the General Motors/University of Michigan Collaborative Research Laboratory in Smart Materials and Structures.

\section{References}

1. Shaw, J.A., Churchill, C.B., and Iadicola, M.A., "Tips and Tricks for Characterizing Shape Memory Alloy Wire: part 1-differential
Scanning Calorimetry \& Basic Phenomena," Experimental Techniques 32(5)::55-62 (2008).

2. Shaw, J.A., and Kyriakides, S., "On the Nucleation and Propagation of Phase Transformation Fronts in a NiTi Alloy," Acta Materialia 45(2):683-700 (1997).

3. Sutton, M.A., Chu, T.C., and Ranson, W.F., "Applications of Digital-image-correlation Techniques to Experimental Mechanics," Experimental Mechanics 25(3):232-244 (1985).

4. Daly, S., Ravichandran, G., and Bhattacharya, K., "Stressinduced Martensitic Phase Transformation in Thin Sheets of Nitinol," Acta Materialia 55(10):3593-3600 (2007).

5. Churchill, C.B., and Shaw, J.A., "Shakedown Response of Conditioned Shape Memory Alloy Wire," Proceedings of the SPIE 15th Annual International Symposium on Smart Structures and Materials 6929:69291F (2008).

6. Iadicola, M.A., and Shaw, J.A., "An Experimental Setup for Measuring Unstable Thermo-mechanical Behavior of Shape Memory Alloy Wire," Journal of Intelligent Material Systems and Structures 13(2):157-166 (2002).

7. Iadicola, M.A., and Shaw, J.A., "Rate and Thermal Sensitivities of Unstable Transformation Behavior in a Shape Memory Alloy," International Journal of Plasticity 20:577-605 (2004).

8. Shaw, J.A., Material Instabilities in a Nickel-titanium Shape Memory Alloy, PhD Dissertation, Department of Aerospace Engineering, The University of Texas at Austin (1997).

9. Shaw, J.A., and Kyriakides, S., "Thermomechanical Aspects of NiTi," Journal of the Mechanics and Physics of Solids 43(8):12431281 (1995)

10. Chang, B.-C., Shaw, J.A., and Iadicola, M.A, "Thermodynamics of Shape Memory Alloy Wire: modeling, Experiments, and Application," Continuum Mechanics and Thermodynamics 18(1-2):83118 (2006).

11. Iadicola, M.A., On Propagating Instabilities in Nickeltitanium and Steel Alloys, $\mathrm{PhD}$ Thesis, University of Michigan (2002)

12. Ananthan, V.S., and Hall, E.O, "Microscopic Shear Bands at Luders Fronts in Mild Steel," Scripta Metallurgica 21(4):519-520 (1987)

13. Kyriakides, S., and Miller, J.E., "On the Propagation of Lüders Bands in Steel Strips," Journal of Applied Mechanics 67:645-653 (2000)

14. Coates, P.D., and Ward, I.M., "Neck Profiles in Drawn Linear Polyethylene," Journal of Materials Science 15:2897-2914 (1980).

15. Vincent, P.I., "The Necking and Cold-drawing of Rigid Plastics," Polymer 1:7-19 (1960).

16. Ericksen, J.L., "Equilibrium of Bars," Journal of Elasticity 5(3-4):191-201 (1975).

17. Gong, L., and Kyriakides, S., "On the Crushing Stress of Open Cell Foams," Journal of Applied Mechanics 73:807-814 (2006).

18. Papka, S., and Kyriakides, S., "In-plane Compressive Response of Crushing of Honeycomb," Journal of the Mechanics and Physics of Solids 42:1499-1532 (1994).

19. Dyau, J.Y., and Kyriakides, S., "On the Propagation Pressure of Long Cylindrical Shells Under External Pressure," International Journal of Mechanical Sciences 35(8):675-713 (1993).

20. Kyriakides, S., and Chang, Y.-C., "The Initiation and Propagation of a Localized Instability in an Inflated Elastic Tube," International Journal of Solids and Structures 27(9):1085-1111 (1991).

21. Kyriakides, S., "Propagating Instabilities in Structures," Hutchinson, J.W., and Wu, T.Y. (eds), Advances in Applied Mechanics, Volume 30, Academic Press, Boston, MA, pp.67-189 (1994). 